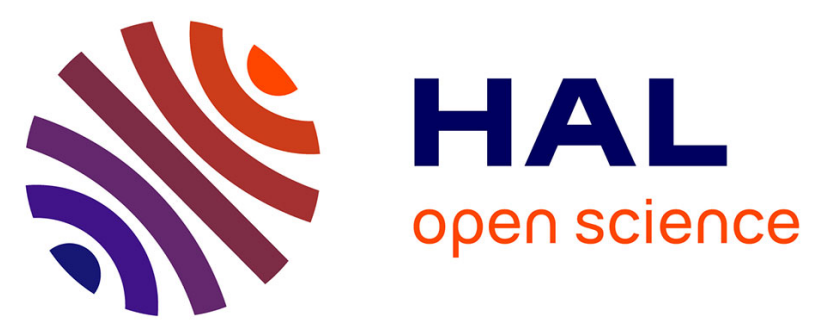

\title{
Sur l'origine morphologique des écoulements par l'analyse d'observations hydrologiques distribuées. Application à deux bassins versants cévenols (Gard, France)
}

D. Maréchal, P.A. Ayral, Jean-Stéphane Bailly, C. Puech, S. Sauvagnargues Lesage

\section{To cite this version:}

D. Maréchal, P.A. Ayral, Jean-Stéphane Bailly, C. Puech, S. Sauvagnargues Lesage. Sur l'origine morphologique des écoulements par l'analyse d'observations hydrologiques distribuées. Application à deux bassins versants cévenols (Gard, France). Géomorphologie: relief, processus, environnement, 2013, 1, p. 47 - p. 62 . hal-00838012

\section{HAL Id: hal-00838012 \\ https://hal.science/hal-00838012}

Submitted on 24 Jun 2013

HAL is a multi-disciplinary open access archive for the deposit and dissemination of scientific research documents, whether they are published or not. The documents may come from teaching and research institutions in France or abroad, or from public or private research centers.
L'archive ouverte pluridisciplinaire HAL, est destinée au dépôt et à la diffusion de documents scientifiques de niveau recherche, publiés ou non, émanant des établissements d'enseignement et de recherche français ou étrangers, des laboratoires publics ou privés. 


\title{
Sur l'origine morphologique des écoulements par l'analyse d'observations hydrologiques distribuées. Application à deux bassins versants cévenols (Gard, France)
}

\section{Morphological origin of run-offs through distributed hydrological observations on two Mediterranean catchments in the Cevennes region (Gard, France)}

\author{
Denis Maréchal*,**, Pierre-Alain Ayral*,***, Jean-Stéphane Bailly****, \\ Christian Puech**, Sophie Sauvagnargues-Lesage*
}

\section{Résumé}

Cet article se propose d'étudier les relations qu'entretient la morphologie des bassins et de leurs réseaux hydrographiques avec les débits survenant lors des crues. Des descripteurs morphologiques issus de l'analyse de modèle numérique de terrain (MNT) par un algorithme original sont confrontés à des mesures de débits effectuées sous épisodes et spatialement distribuées à l'intérieur de deux bassins expérimentaux situés au sein du bassin du Gardon d'Anduze (Gard). Les résultats montrent l'influence des surfaces drainées sur les débits mesurés et font apparaître deux types de fonctionnement des réseaux hydrographiques : un fonctionnement de type réseau principal, dans lequel les débits observent une relation puissante avec les surfaces drainées, et un fonctionnement de type réseau secondaire, dans lequel les débits ne peuvent être expliqués ni par la surface drainée ni par les descripteurs morphologiques étudiés. Ces résultats font ainsi apparaître la distinction entre des fonctionnements de type versant et de type réseau. Ils mettent également en avant les différences entre les deux bassins expérimentaux étudiés, notamment la variabilité spatiale des deux types de fonctionnements et l'influence prédominante de la géologie et de la pédologie des sites sur les mécanismes de mise en eau des réseaux hydrographiques.

Mots clés : morphologie, observations hydrologiques distribuées, fonctionnements versants/réseaux, Gardon d'Anduze.

\begin{abstract}
This article is aimed at studying relations between basin and hydrological network morphologies and flood discharges. Morphological traits extracted from the Digital Elevation Model (DEM) using an original algorithm are used to explain spatially distributed rainfall discharges on two experimental catchments within the Gardon d'Anduze river basin (Gard). Results show the influence of drained areas on discharge measures and highlight two types of hydrological behaviour: a principal network where discharge measures follow a power-law function with drained areas and a secondary network where discharge measures can be neither explained by drained area values nor by morphological traits extracted from the DEM. This result emphasises the distinction between slopes and networks hydrological behaviours. Moreover, it points out the differences between the two catchment studied, particularly on the spatial variability of the both types function and reveals the most prominent geological and pedological influence on the mechanisms which are responsible for the hydrological activity of catchment networks.
\end{abstract}

Key words: morphology, spatially distributed hydrological measures, slopes/network functioning, Gardon d'Anduze catchment.

\section{Abridged English version}

This paper tries to evaluate morphological influence on the production of surface run-offs in natural ungauged catchments in the Mediterranean region. The goal is to analyse the type of relation between morphological descriptors of hydrological networks and catchments and their hydrological responses under rainfall episodes. Is the relation unique and stable in space and time? Is it scale dependent? Does it correspond to distinct hydrological functioning?

\footnotetext{
* Centre de recherche LGEI - Ecole des Mines d'Alès - France (dens.marechal@gmail.com).

** CEMAGREF - UMR TETIS - Montpellier - France.

*** UMR ESPACE 6012 CNRS - Nice - France.

**** AgroParisTech - UMR TETIS-UMR LISAH - Montpellier - France.
} 
Two experimental catchments situated in the Gardon d'Anduze river basin (Cartaou and Crébadès) were monitored during rainfall episodes (between 2007 and 2010), by systematic surveys with observation points distributed on each of their barren gullies. Discharge measures and qualitative observations allowed detailed hydrological responses to be collected. Not only run-off intensities and localisations but also run-offs stability were first mapped and then analysed regarding terrain morphological features.

An original algorithm, developed by D. Maréchal et al. (2011), provides the morphological characteristics of hydrological networks and basins. These features, extracted from TIN DEM, were then used to analyse morphological influence on hydrological responses observed under rainfall conditions.

The results show an insignificant influence of chosen morphological index (tab. 5) on flood responses in all parts of the studied catchments. Drained area seems to be a relevant feature to model catchment discharges on the main reaches of the whole hydrographical network. Indeed, power-law functions which link drained area and measured discharges are only confirmed for drained area values superior to a certain threshold, stable in the case of the Cartaou catchment $\left(4000 \mathrm{~m}^{2}\right)$ and variable for the Crébadès, depending on the observed episodes (fig. 4). This drained area threshold highlights two types of hydrographical networks and behaviours: principal networks where discharges are driven by hydraulic properties and secondary networks where hydrological behaviour is related to slopes function, depending on soil and substratum properties.

Relations between run-off stability (which gives occurrences of water presence in networks across all of the observed episodes) and morphological features are also hard to establish for the whole network of the studied catchments. Although the principal network is the most perennial one on the Crébadès catchment, the relation between run-off stability and affiliation to the principal network is not clear on the Cartaou. This observation shows that if principal network discharges are influenced by hydraulic conditions, they do not seem to play a role in the frequency of water presence.

This paper has permitted an approach to quantitative evaluation of a relevant feature in hydrological modelling: the threshold separating two types of hydrological functioning: production (slopes) and transfer (networks). This consequently has a significant impact for modelling discharges in ungauged catchments, using simple features extracted from a DEM.

Moreover, it has confirmed the different behaviours of the two studied catchments. Water occurrence and extension of principal networks show a more hydrologically reactive catchment with a more temporary network in the Crébadès watershed, mostly due to the type of substratum (shale) and the amount of fracturing. It is more slope dependent and runoffs can be seen at very high altitudes. The Cartaou catchment presents more perennial networks and a greater extension of principal networks. It is more drained area dependent and surface run-offs in altitude are fewer. Once again, this result is in adequation with the more permeable type of substratum of this catchment: granite arenas.
In the end, this study shows the interest of crossing geomorphological features extracted from the DEM (stable in time and relevant for hydrological modelling) with variable hydrological data (quantitative and qualitative) spatially distributed inside experimental catchments, in order to understand temporal and spatial variations of the hydrological responses to rainfall episodes.

\section{Introduction}

Quelle que soit leur origine, les écoulements de surface sur les bassins versants tendent à se concentrer de l'amont vers l'aval, sous l'effet des gradients horizontaux de potentiel gravitaire. Leur vitesse augmente avec la pente du versant mais diminue avec la rugosité de la surface (Moore et Foster, 1990). L'écoulement tend donc à se concentrer vers l'aval, d'abord dans un chevelu convergeant de rigoles puis dans un réseau de thalwegs que le ruissellement contribue à maintenir et à développer par érosion hydrique. Il peut cependant diverger ou s'anastomoser là où la pente diminue, dans des zones de divergence des flux, en fonction de la topographie locale. Une importante redistribution spatiale de l'eau de surface résulte de cette hiérarchisation progressive du ruissellement le long d'un versant. Le long du réseau hydrographique, l'intensité des écoulements varie fortement de l'amont vers l'aval, en fonction des apports latéraux venant des versants et par des échanges nappes-rivières. Il peut varier de manière brutale et discontinue, augmentant à chaque confluence et sources, diminuant à chaque diffluence (Ambroise, 1998).

L'influence de la morphologie du réseau hydrographique, assurant la fonction de transfert des volumes d'eau jusqu'à l'exutoire d'un bassin, a été relevée sur les hydrogrammes par de nombreux auteurs depuis la moitié du $\mathrm{XX}^{\mathrm{e}}$ siècle (Moussa, 2009). Ainsi, depuis les travaux de R.E. Horton (1932) sur la classification des réseaux selon la topologie des différents drains, de nombreux auteurs se sont penchés sur l'analyse quantitative des réseaux pour étudier l'influence de leur agencement et de leurs caractéristiques physiques (longueur, pente, largeur, microtopographie) sur la forme de l'hydrogramme enregistré à l'exutoire des bassins étudiés (Rodriguez-Iturbe et Valdès, 1979 ; Gupta et al., 1980 ; White et al., 2004 ; Kumar et al., 2007 ; Singh et al., 2007 ; Bhadra et al., 2008 ; Lee et al., 2008 ; Moussa, 2009), pour prévoir des pics de crue (Mantilla et al., 2006 in Moussa, 2009) ou pour établir des lois d'échelles pour les débits moyens annuels (De Vries et al., 1994). A.B. White et al. (2004) ont notamment montré que la réponse hydrologique est fortement gouvernée par la structure du réseau hydrographique. D'après cette étude menée sur une variété de bassins versants de l'Illinois, les caractéristiques physiques des réseaux (profondeur, section mouillée, etc.), gouvernant notamment les vitesses de propagation de l'onde de crue, sont d'une influence négligeable devant l'agencement spatial (topologie) des différents drains les composant. Les auteurs insistent sur l'intérêt de l'utilisation des indices géomorphologiques capables de synthétiser les informations utiles à la modélisation hydrologique, en utilisant des para- 
mètres extraits de l'analyse de modèles numériques de terrain $(\mathrm{MNT})$.

Le bassin n'est plus considéré aujourd'hui comme représentant une entité unique et homogène mais comme l'assemblage complexe d'unités fonctionnelles particulières dont les échanges sont gouvernés par des caractéristiques hydrogéomorphologiques locales. La seule analyse de l'hydrogramme à l'exutoire ne suffit pas à expliquer la variabilité des réponses hydrologiques intra-bassin, notamment pour des bassins soumis à des précipitations particulièrement violentes. Dans ce contexte et pour des bassins soumis à d'intenses événements pluvieux (i.e., crues éclair), existe-til toujours une relation univoque et forte entre l'intensité et la distribution des écoulements surfaciques et la morphologie des réseaux et des bassins ? Si oui, jusqu'à quelle échelle ? Et quelle est la stabilité de cette relation à l'intérieur d'un bassin?

En utilisant un dispositif expérimental original d'observation des écoulements, varié et spatialement distribué, le présent travail 1) étudie les variations spatio-temporelles des écoulements observés sous différents épisodes pluvieux en de multiples points des réseaux hydrographiques en eau et 2) cherche à identifier les processus principaux, notamment ceux résultant de la morphologie des bassins, qui pourraient expliquer la dynamique des écoulements. Les objectifs sont donc ici de confronter les indices morphologiques des réseaux avec les observations hydrologiques distribuées, ceci sur deux bassins versants expérimentaux situés dans les Cévennes (Gard, France). Cette confrontation permettra d'étudier l'influence de la morphologie sur les écoulements au sein des réseaux mais également d'approcher les limites dynamiques entre un fonctionnement de type versant (production) et réseau (transfert) au sein d'un bassin-versant. Après une présentation des bassins pilotes, de la stratégie expérimentale et des descripteurs morphologiques utilisés, les résultats d'analyse de la corrélation entre descripteurs morphologiques et réponses hydrologiques seront présentés en s'attachant plus particulièrement aux débits et aux occurrences de mise en eau des drains sur les deux bassins expérimentaux.

\section{Présentation des bassins expérimentaux}

\section{Le site d'étude}

Les deux bassins versants expérimentaux du Cartaou (commune de Peyrolles) et du Crébadès (commune de Tourgueille) sont situés dans le bassin-versant du Gardon d'Anduze (Martin et al., 2008). Ce dernier est localisé dans la partie nordouest du département du Gard (fig. 1).

D'une superficie de $545 \mathrm{~km}^{2}$, le bassin-versant du Gardon d'Anduze est régulièrement soumis à des « crues éclair » et a fait l'objet de très nombreuses études hydrologiques généralement orientées sur la prévision de ce type de phénomè- ne (Moussa, 1991 ; Gaume et Bouvier, 2004 ; Delrieu et al., 2005 ; Ayral et al., 2005 ; Marchandise, 2007 ; Toukourou et al., 2009 ; Maréchal, 2011 ; Tramblay et al., 2011). La géologie de ce bassin versant est essentiellement composée de schistes qui représentent environ $65 \%$ de sa superficie totale. Les granites, concentrés dans la partie intermédiaire du bassin, représentent quant à eux $15 \%$ de la superficie totale du bassin. Enfin, des formations sédimentaires (calcaires, dolomies, grès) sont présentes dans la partie aval du bassin, constituant $20 \%$ de sa superficie totale (Ayral, 2005). Une des caractéristiques principales de ce bassin réside dans le gradient d'altitude entre son point culminant et son exutoire, de l'ordre de $1000 \mathrm{~m}$ (Ayral, 2005). Il est entaillé de vallées linéaires transversales, sub-parallèles avec des pentes prononcées (Séranne et al., 2001). Dominées par des crêtes étroites aux pentes escarpées et ravinées (i.e., les « serres cévenoles »), les vallées longues et encaissées sont représentatives des paysages observés dans ce bassin. Sur des versants en pente forte, très érodés, les sols sont peu épais et la roche affleure souvent (Martin et al., 2008). Du point de vue climatique, le bassinversant du Gardon d'Anduze est situé dans le domaine méditerranéen, caractérisé par des étés chauds et secs et des hivers plus frais et humides. Les mois d'été sont les plus secs mais peuvent cependant connaître des événements exceptionnels tels que des orages violents accompagnés de pluies brutales. Pour autant, l'automne est la période où les cumuls et les intensités de pluie sont les plus forts, apportant environ les deux tiers du total annuel des précipitations. Au cours de ces événements qualifiés de « cévenols », les précipitations journalières peuvent dépasser les $400 \mathrm{~mm}$ (Martin et al., 2008).

Les principales caractéristiques des deux bassins versants expérimentaux étudiés sont présentées dans le tab. 1. Le bassinversant du Crébadès est situé dans une zone de schistes sériciteux (Martin, 2006) tandis que le bassin-versant du Cartaou présente un substratum granitique caractéristique de la zone aval du bassin-versant du Gardon d'Anduze. Le bassin-versant du Crébadès présente des sols de type ranker sous couvert forestier situés sur des schistes altérés. Ces derniers sont peu profonds, ne variant que de $30 \mathrm{~cm}$ à $50 \mathrm{~cm}$ du haut vers le bas du versant. Ces sols, très riches en matière organique (supérieure à $20 \%$ ), se caractérisent par une densité apparente très faible et une porosité importante (Ayral, 2005). Au niveau des écoulements, cela implique un fort potentiel de circulation rapide de sub-surface, circulation favorisée par l'importance de la pente

\begin{tabular}{|l|c|c|}
\hline \multicolumn{1}{|c|}{ Bassins versants } & Cartaou (Tourgueille) & Crébadès (Valescure) \\
\hline Surface $\left(\mathrm{en} \mathrm{km}^{2}\right)$ & 0,6 & 0,9 \\
\hline Pente moyenne à l'exutoire (en \%) & 49 & 65 \\
\hline Altitude maximum (en m) & 740 & 1220 \\
\hline Altitude minimum (en m) & 300 & 740 \\
\hline
\end{tabular}

Tab. 1 - Caractéristiques des bassins versants expérimentaux.

Tab. 1 - Experimental catchments characteristics. 


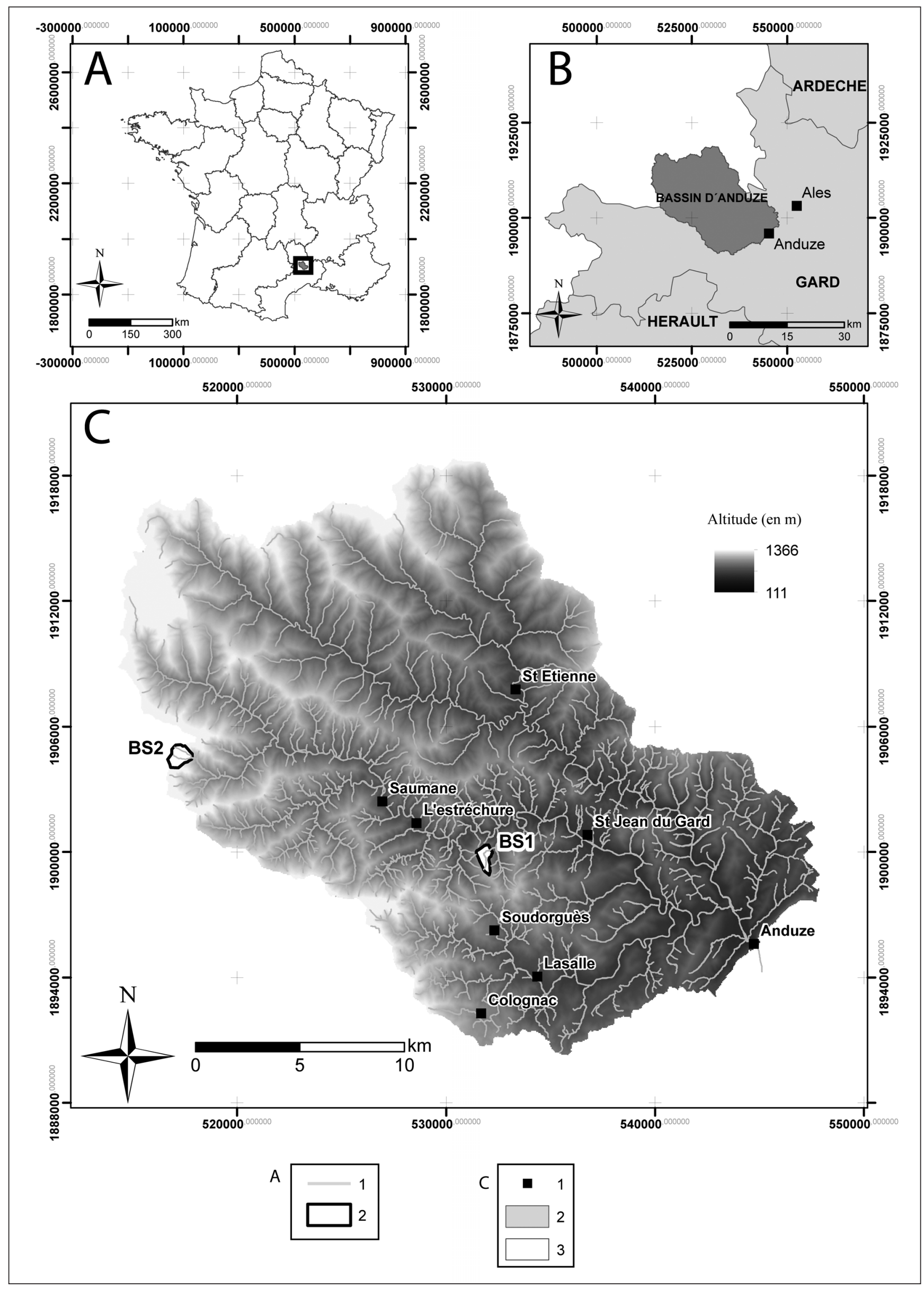


Fig. 1 - Localisation du bassin-versant du Gardon d'Anduze et des bassins versants expérimentaux. $A$ : Situation géographique du bassin du Gardon d'Anduze en France (dans l'encadré noir). 1 : principaux cours d'eau ; 2 : bassins pilotes. B : Départements inclus dans le bassin du Gardon d'Anduze. C : Le bassin du Gardon d'Anduze. 1 : villes ; 2 : départements ; 3 : régions ; $B S 1$ : bassin-versant du Valescure ; BS2 : bassin-versant de la Lieure.

Fig. 1 - Location of the Gardon d'Anduze catchment and experimental catchments. A: Localisation of the Gardon d'Anduze catchment in France (in the black square). 1: main rivers; 2: experimental catchments. B: Departements of the surrounding of the Gardon d'Anduze catchment. C: The Gardon d'Anduze catchment. 1: cities; 2: departments (administrative French subdivision); 3: region (administrative French subdivision); BS1: Valescure catchment ; BS2: Lieure catchment.

et de la macroporosité. Le bassin du Cartaou présente la même configuration de sol, ici de type ranker sur granite dont les horizons A sont peu épais, très caillouteux, riches en matière organique, de densité apparente faible et de porosité élevée. Ces rankers surmontent une arène et des roches altérées de manière hétérogène sur le bassin (Ayral et al., 2005). La perméabilité de ces arènes favorise les écoulements profonds et joue probablement un rôle de stockage des eaux précipitées. Cependant, l'hétérogénéité spatiale de ces formations sur le bassin, la présence de fortes pentes sur les versants et la macroporosité des sols favorisent, comme dans le bassin-versant du Crébadès, des écoulements de sub-surface, voire localement de surface (Martin, 2006) dans les zones de saturation du profil.

Soumis aux mêmes contraintes climatiques, la position relative à l'intérieur du bassin du Gardon d'Anduze entraîne cependant des différences entre les deux bassins étudiés. Le bassin du Crébadès, à la limite nord-ouest du bassin du Gardon d'Anduze, est situé plus en altitude. Soumis aux influences atlantiques d'ouest, ses versants aux fortes pentes sont également susceptibles de bloquer les perturbations méditerranéennes. Cette double exposition climatique peut entraîner des précipitations plus importantes que dans le bassin du Cartaou. De plus, on y observe des températures plus fraîches et une couverture neigeuse plus persistante en hiver. Majoritairement dominé par des résineux (pins, douglas), des châtaigniers, mais aussi par de la végétation de garrigue (genêts, bruyères...) sur les versants sud, même en altitude, le bassin du Crébadès possède également des hêtraies, typiques des altitudes supérieures à 1000 m. Dans le bassin-versant du Cartaou, au centre du bassin du Gardon d'Anduze, les influences climatiques sont davantage méditerranéennes. Les espèces dominantes sont le châtaignier et le chêne vert. Quelques résineux y sont plantés et l'on retrouve également de la végétation de garrigue. Cependant aucune essence d'altitude n'est présente

Tab. 2 - Indice d'écoulement et intensité des écoulements observés.

Tab. 2 - Runoff index and corresponding intensity of the observed runoff. dans ce deuxième bassin. L'ensemble de ces différences peut avoir des conséquences importantes sur les mécanismes et les réponses hydrologiques observés sous épisodes. Toutefois, les fortes pentes de ces deux bassins, même s'ils présentent des substratums différents, ont tendance à favoriser les écoulements sub-surfaciques.

\section{Stratégie expérimentale}

Les difficultés rencontrées dans l'étude des réponses hydrologiques des bassins versants aux précipitations sont liées à l'importance des variations spatio-temporelles des processus hydrologiques au sein des différentes entités constituant un même bassin. Si au départ les hydrologues considéraient le bassin-versant comme une unité homogène, il est aujourd'hui compris comme un agencement complexe d'entités dans lesquelles se déroulent des processus hydrologiques variés participant de manière intermittente à la production d'écoulements de surface et au soutien des débits (Ambroise, 1998). Une des possibilités d'étudier les variations spatio-temporelles de ces différents processus est de spatialiser le suivi des réponses hydrologiques à l'intérieur même des bassins, lors des différents épisodes pluvieux auxquels ils sont soumis, afin de rendre compte de la diversité des réponses hydrologiques et de leurs variations spatio-temporelles. C'est pourquoi l'approche suivie dans cette étude s'appuie sur des données acquises en différents points d'observation et de mesure, distribués à l'intérieur même des bassins, sous épisodes et sur l'ensemble du réseau hydrographique en eau. Elle permet de caractériser les débits instantanés, d'observer les écoulements surfaciques, de localiser le lieu de leur naissance et d'évaluer l'importance de l'extension du réseau hydrographique en eau. L'intérêt de cette approche est de multiplier les points de mesure à l'intérieur de chaque bassin afin d'obtenir une vision globale et spatialisée des dynamiques hydrologiques sous épisodes.

\begin{tabular}{|c|l|}
\hline $\begin{array}{c}\text { Indices } \\
\text { d'écoulement }\end{array}$ & \multicolumn{1}{|c|}{ Intensités des écoulements observés } \\
\hline 0 & Pas d'écoulement \\
\hline 1 & Présence d'humidité \\
\hline 2 & Suintements \\
\hline 3 & Ecoulement trop faible pour la mesure \\
\hline 4 & $\begin{array}{l}\text { Ecoulement faible }(\leq 1 \mathrm{~L} / \mathrm{s}) \text { dont la mesure n'a pas été } \\
\text { techniquement possible }\end{array}$ \\
\hline 5 & $<1 \mathrm{~L} / \mathrm{s}$ \\
\hline 6 & $1-5 \mathrm{~L} / \mathrm{s}$ \\
\hline 7 & $5-10 \mathrm{~L} / \mathrm{s}$ \\
\hline 8 & $10-20 \mathrm{~L} / \mathrm{s}$ \\
\hline 9 & $20-50 \mathrm{~L} / \mathrm{s}$ \\
\hline 10 & $50-100 \mathrm{~L} / \mathrm{s}$ \\
\hline 11 & $100-200 \mathrm{~L} / \mathrm{s}$ \\
\hline 12 & $>200 \mathrm{~L} / \mathrm{s}$ \\
\hline
\end{tabular}




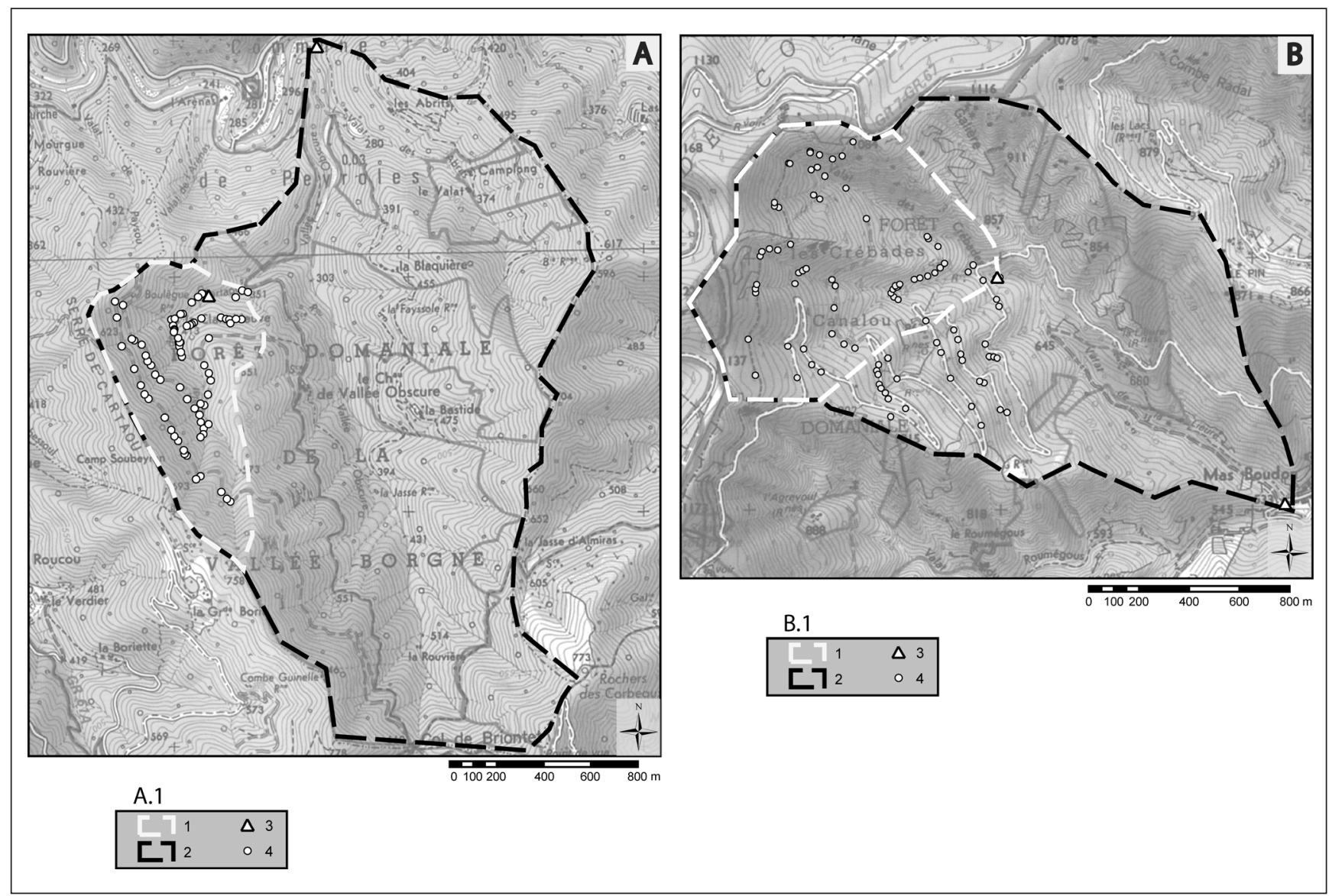

Fig. 2 - A : Bassins expérimentaux de la vallée BorgneA.1 : dispositif de suivi hydrologique des bassins versants emboités de la vallée Borgne (bassin du Valescure : $3,88 \mathrm{~km}^{2}$; bassin du Cartaou : 0,63 $\mathrm{km}^{2}$ ). 1 : bassin-versant du Cartaou ; 2 : bassin-versant du Valescure ; 3 : stations limnimétriques ; 4 : points d'observation. B : Bassins emboités de la Lieure et du Crébadès ; B.1 : dispositif de suivi hydrologique des bassins versants emboités de la commune de Tourgueille (bassin de la Lieure $: 2,58 \mathrm{~km}^{2}$; bassin du Crébadès :0,88 $\mathrm{km}$ ).

Fig. 2 - A: Experimental catchments of the Borgne valley; A.1: device for hydrological monitoring of the embedded catchments of the Borgne valley (Valescure catchment: 3.88 km²; Cartaou catchment: 0.63 km²). 1: Cartaou catchment; 2: Valescure catchment; 3 : limnimetric stations; 4: measurement. B: Nested catchments of the Lieure and Crébadès; B.1: device for hydrological monitoring of the embedded catchments of Tourgueille city (Lieure catchment: $2.58 \mathrm{~km}^{2}$; Crébadès catchment: $0.88 \mathrm{~km}^{2}$ ). 1: Crébadès catchment; 2: Lieure catchment; 3: limnimetric stations; 4: measurement.

\section{Méthodologie expérimentale}

Un suivi des réactions des cours d'eau et des ravines a été mis en place dans les deux bassins versants expérimentaux. Un inventaire exhaustif des drains a été effectué et des points d'observation ont été identifiés en leur sein (fig. 2). Les campagnes de mesure hydrologique (i.e., estimations de débit) pendant et/ou après un événement pluvieux majeur ont été réalisées à ces points d'observation. Pour des raisons d'incertitude temporelle de l'apparition des phénomènes météorologiques, certaines observations ont pu être réalisées sous épisodes tandis que d'autres ont été effectuées dans les heures ou les jours suivant le pic de crue. En outre, des observations ont été menées en période intermédiaire (i.e., sans pluie majeure), dans le but d'établir l'état hydrologique des bassins avant épisode. Pendant les campagnes de terrain, pour chacun des points d'observation, lorsqu'un écoulement continu était mesurable, des jaugeages ont été effectués par empotement ou par dilution, en fonction de l'importance du débit et de la configuration locale du drain (Maréchal et al., 2009 ;
Maréchal, 2011). Des estimations de débit maximum ont pu également être réalisées à partir de la hauteur des laisses de crue en utilisant la formule de Manning-Strickler (Gaume, 2002). Lorsque le débit n'était pas directement mesurable, des observations qualitatives sur l'intensité des écoulements ont été effectuées. Ces observations et les débits mesurés ont ensuite été ordonnés sous la forme d'indices d'écoulement, présentés dans le tab. 2.

\section{Nature des informations hydrologiques suivies}

Le tab. 2 présente les données recueillies sur le terrain et qui seront utilisées dans la suite de cette étude. Durant la période d'observation (2008-2010), huit campagnes de mesures ont été réalisées, dont quatre durant des événements de crue d'automne ou de fin d'hiver et quatre en période intermédiaire. Le tableau 3 présente les caractéristiques de 


\begin{tabular}{|l|l|l|}
\hline \multicolumn{1}{|c|}{ Caractéristiques } & \multicolumn{1}{|c|}{ Méthode } & \multicolumn{1}{c|}{ Données utilisées } \\
\hline $\begin{array}{l}\text { Débits ou intensités des écoulements } \\
\text { surfaciques }\end{array}$ & $\begin{array}{l}\text { Mesure par dilution, par emportement, } \\
\text { observations }\end{array}$ & Indices d'écoulement \\
\hline Origine des écoulements surfaciques & $\begin{array}{l}\text { Localisation le long des réseaux de } \\
\text { la naissance des écoulements surfaciques }\end{array}$ & $\begin{array}{l}\text { Cartographie des points de départs } \\
\text { des écoulements surfaciques }\end{array}$ \\
\hline $\begin{array}{l}\text { Extension des réseaux hydrographiques } \\
\text { en eau }\end{array}$ & $\begin{array}{l}\text { Synthèse des données spatialisées sur } \\
\text { les écoulements }\end{array}$ & $\begin{array}{l}\text { Cartographie des réseaux en eau } \\
\text { Détermination des occurrences de mise } \\
\text { en eau des réseaux suivis }\end{array}$ \\
\hline
\end{tabular}

Tab. 3 - Données recueillies sur le terrain.

Tab. 3 - Data collected on the field.

ces événements. La combinaison des données récoltées sous épisodes et en périodes intermédiaires a permis d'obtenir une image de la situation hydrologique du réseau hydrographique dans chacun des bassins en fonction des épisodes, permettant par la suite l'analyse des processus événementiels responsables des réponses observées.

\section{Descripteurs morphologiques}

L'extraction des réseaux et des caractéristiques hydromorphologiques des deux bassins expérimentaux a été réalisée avec l'algorithme NET (network extraction from TIN ; Maréchal et al., 2010 ; Maréchal, 2011). Basée sur un MNT vecteur triangulé (triangulated irregular network : TIN), cette méthode originale permet d'attribuer à chacun des drains et des points d'observation des caractéristiques hydromorphologiques telles que la surface drainée, la pente locale ou la pente moyenne amont. Cette méthode présente deux approches originales : 1) une décomposition de chaque facette triangulaire du TIN en quatre sous-facettes afin de permettre le transit des eaux de surface soit à travers les triangles initiaux, soit le long de leurs arêtes en fonction de la morphologie locale ; 2) une détection automatique des points de départ des réseaux de thalwegs, en considérant que ce réseau ne peut être défini que par la présence d'un thalweg détectable sur le MNT (Maréchal, 2011). L'algorithme NET fourni un ensemble de descripteurs de la morphologie des bassins et des réseaux hydrographiques extraits (tab. 4). Dans la suite de ce travail seront uniquement présentés les descripteurs qui ont montré un intérêt particulier pour expliquer les débits mesurés sous épisodes.

\begin{tabular}{|c|c|c|c|c|c|c|}
\hline $\begin{array}{l}\text { Bassins } \\
\text { versants }\end{array}$ & Date & $\begin{array}{l}\text { Débit maximum } \\
\quad(e n \mathrm{~L} / \mathrm{s})\end{array}$ & $\begin{array}{l}\text { Cumul jour } \\
\text { (en mm) }\end{array}$ & $\begin{array}{c}\text { IPA } \\
\text { (en mm) }\end{array}$ & $\begin{array}{c}\text { Intensité maximum } P \\
(\text { en } \mathrm{mm} / \mathrm{h})\end{array}$ & Observations \\
\hline \multirow{8}{*}{ Cartaou } & 03/02/2009 & 710 & 80 & 255 & 12 & Pendant l'épisode \\
\hline & 23/10/2008 & 450 & 0 & 160 & 110 & Pendant l'épisode \\
\hline & $22 / 02 / 2010$ & 100 & 30 & 160 & 10 & Pendant l'épisode \\
\hline & $23 / 10 / 2009$ & 65 & 0 & 160 & 50 & Pendant l'épisode \\
\hline & $12 / 02 / 2010$ & 35 & & 70 & & Intermédiaire \\
\hline & $28 / 05 / 2009$ & 5 & & 10 & & Intermédiaire \\
\hline & $29 / 07 / 2008$ & 2 & & 6 & & Intermédiaire \\
\hline & 28/08/2009 & 0,5 & & 7 & & Intermédiaire \\
\hline \multirow{7}{*}{ Crébadès } & $02 / 11 / 2008$ & 880 & 120 & 360 & 60 & Pendant l'épisode \\
\hline & $22 / 10 / 2008$ & 100 & 30 & 40 & 50 & Pendant l'épisode \\
\hline & 29/07/2008 & 30 & & & & Intermédiaire \\
\hline & 29/05/2009 & 15 & & & & Intermédiaire \\
\hline & 08/09/2010 & 8 & & & & Intermédiaire \\
\hline & 03/09/2009 & 4 & & & & Intermédiaire \\
\hline & $12 / 02 / 2009$ & N.d & 0 & 450 & 0 & $2 \mathrm{j}$ après l'épisode \\
\hline
\end{tabular}

Tab. 4 - Evénements observés dans les bassins versants du Cartaou et du Crébadès. IPA : indice des précipitations antérieures défini par P. Chevalier (1983) et calé dans le bassin-versant du Gardon d'Anduze par A. Marchandise (2007). P : intensité calculée sur un pas de temps de 5 min. N.d : données non disponibles.

Tab. 4 - Observed episodes in Cartaou and Crebades catchments. IPA: index for anterior precipitation as defined by P. Chevalier (1983) and evaluated in the Gardon d'Anduze catchment by A. Marchandise (2007). P: precipitation intensity calculated on a 5 min step. N.d: nonavailable data. 


\section{Surface amont drainée}

Elle est l'une des données principalement utilisée en hydrologie, en géomorphologie et pour l'étude du transport de sédiments. La surface contribuant aux flux observés en un point donné est particulièrement utilisée pour déterminer les zones de saturation et/ou d'exfiltration des flux par excès de saturation (Tarboton, 1997). Pour L.W. Martz et J. Garbrecht (1995), sous l'hypothèse généralement admise que les écoulements sont générés de manière uniforme et que la réponse géomorphologique et érosive à ces écoulements est spatialement homogène sur l'ensemble d'un bassin, la seule surface amont drainée suffit à rendre compte des interactions complexes qu'entretiennent la géologie, la végétation, les sols et la topographie, responsables de la création et de la pérennité de la morphologie des réseaux chenalisés. C'est pourquoi la détermination précise de la surface amont drainée, en chacun des points des bassins suivis, est essentielle à la bonne compréhension des processus hydrologiques ayant cours dans un bassin-versant, notamment lors d'épisodes pluvieux.

\section{Pente locale}

Elle est attribuée ici à des arêtes d'un modèle triangulé de terrain (TIN) suivant la formulation suivante :

$$
p=\frac{(z A-z B)}{\sqrt{\left((x A-x B)^{2}+(y A-y B)^{2}\right)}}
$$

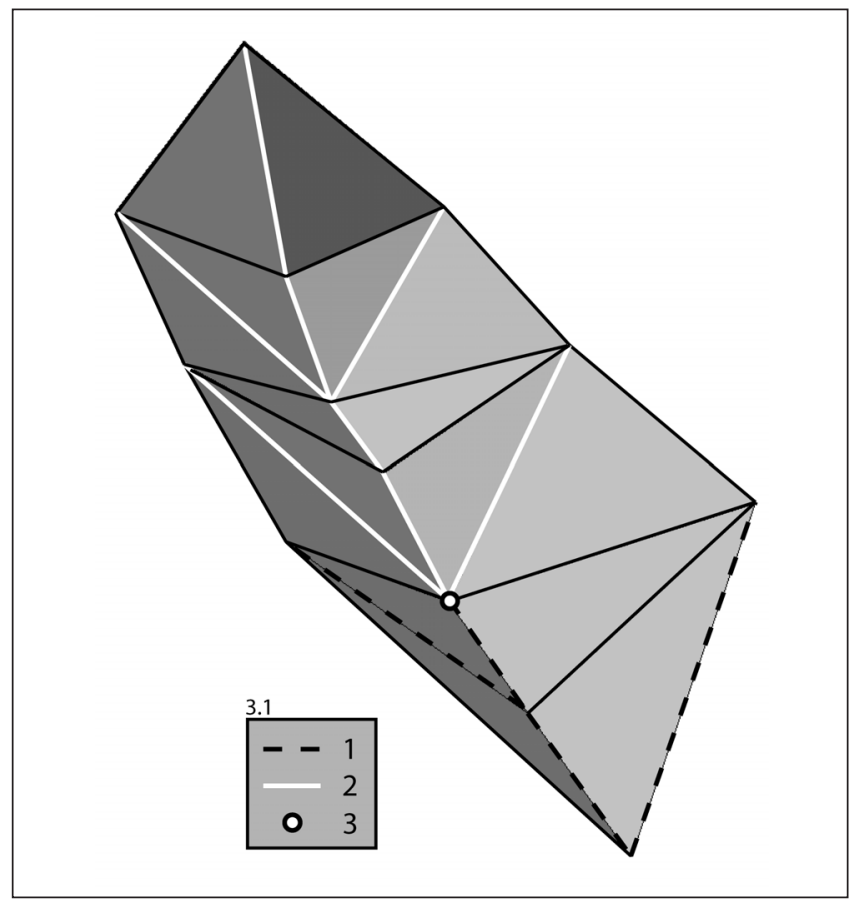

Fig. 3 - Calcul de l'indice de pente moyenne amont. 3.1 : description des symboles utilisés. 1 : arêtes "réseau " situées à l'aval $(\mathrm{Av}) ; 2$ : arêtes « réseau » situées à l'amont $(\mathrm{Am}) ; 3$ : point considéré. Indice de pente moyenne amont: Pma= moyenne [pentes(Am)].

Fig. 3-Mean upward slope calculations. 3.1: description of symbols. 1: downstream "network" edge (Av); 2: upstream "network" edge (Am); 3: considered point. Upstream mean-slope index: Pma= mean [slope $(A m)]$. avec $A=$ nœud de l'arête de plus haute altitude, $B=$ nœud de l'arête de plus faible altitude et $x, y, z=$ coordonnées des nœuds. Il convient de noter que ce mode de calcul de la pente donne des résultats sensibles à la taille des triangles TIN (effet d'échelle).

\section{Indice de pente moyenne amont}

Dans le but d'évaluer l'importance de la pente moyenne du bassin drainé en chacun des points du MNT TIN utilisé, un indice de pente moyenne amont est calculé selon l'exemple proposé in fig. 2. Elle présente le réseau extrait superposé au MNT triangulé (TIN) et le détail du calcul de l'indice de pente moyenne amont. Il s'agit de calculer la moyenne des pentes locales de chacune des arêtes du TIN situées à l'amont du point considéré. Cette moyenne simple fournit ainsi un indicateur de la pente moyenne du bassin drainé au niveau de chacun des nœuds du maillage TIN. Cet indice sera utilisé par la suite pour étudier l'influence de la pente moyenne des bassins drainés sur les réponses hydrologiques observées à leurs exutoires. La détermination de ces trois paramètres a été réalisée à partir d'un TIN issu de nuages de points LIDAR (light detection and ranging) avec une densité moyenne de 5000 points par $\mathrm{km}^{2}$, correspondant à la résolution des MNT qu'il est possible d'obtenir à partir des images satellitales à très haute résolution spatiale disponibles actuellement.

\section{Résultats}

Le suivi hydrologique spatialisé développé dans le cadre de ces travaux a permis de proposer une cartographie des débits et des occurrences de mise en eau des drains dans les deux bassins versants expérimentaux. Il s'agit donc, ici, de confronter ces résultats avec les indicateurs morphologiques extraits de l'algorithme NET, d'abord avec les débits puis avec les occurrences de mise en eau des drains.

\section{Analyse débits observés - surfaces drainées}

La fig. 3 présente les indices d'écoulement observés pour chaque événement suivi (tab. 2) en fonction de la surface amont drainée aux points d'observation dans les bassins versants du Cartaou et du Crébadès. Les débits mesurés sont également présentés en fonction de la surface drainée, événement par événement.

Fig. 4 - Indice d'écoulement, débits et surfaces amont drainées. A : Indices d'écoulement et surfaces amont drainées sur le bassin du Cartaou. B : Débits mesurés et surfaces amont drainées pour différents épisodes observés sur le bassin du Cartaou. C : Indices d'écoulement et surfaces amont drainées sur le bassin du Crébadès, et débits mesurés en fonction de la surface drainée pour l'épisode du 3 novembre 2008.

Fig. 4 - Runoff index, discharges and drained area. A: Runoff index and drained areas on Cartaou catchment. B: Discharges and drained areas for several episodes on Cartaou catchment. C: Runoff index and drained areas on Crébadès catchment and discharges and drained areas for the episode of the 3th November 2008. 


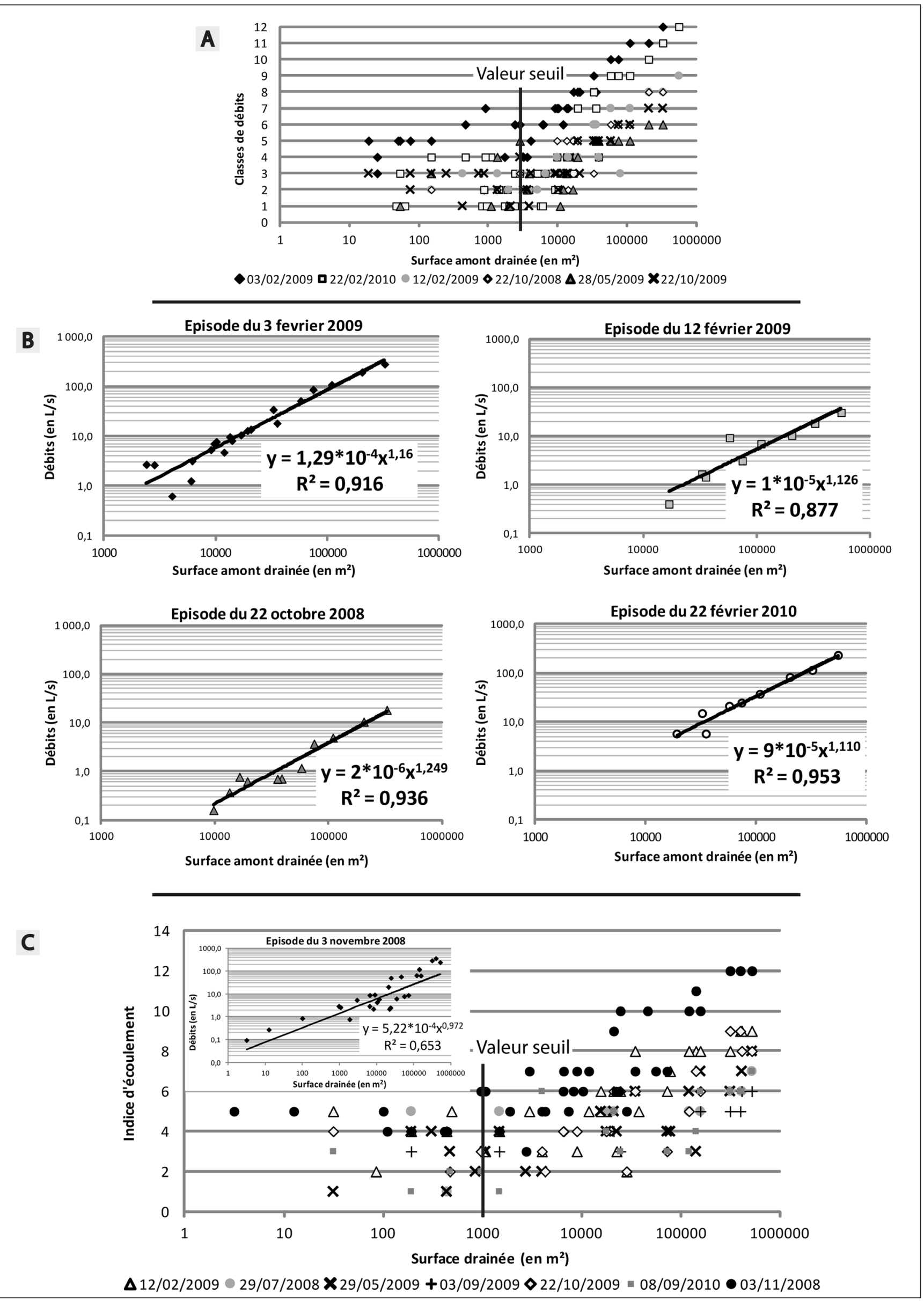


- L'influence de la surface amont drainée sur l'importance des indices d'écoulement observés est clairement mise en évidence dans les deux bassins versants. Les mêmes tendances sont observées si l'on ne considère plus que les débits mesurés. Il convient de noter que la totalité des débits mesurés sont représentés sur les graphiques présentés sur la fig. 3.

- Pour chaque épisode, les débits du Cartaou semblent fortement corrélés à la surface amont drainée. En effet, une relation de type puissance unissant les débits mesurés aux surfaces drainées est observable pour l'ensemble des épisodes à partir d'un seuil surfacique d'environ $5000 \mathrm{~m}^{2}$. Ce seuil, en dessous duquel la relation n'est pas clairement définie, a été déterminé à partir des indices d'écoulement puisqu'ils permettent de prendre en compte l'ensemble des points de mesure ayant réagi aux précipitations événementielles. Sa valeur est d'ailleurs confirmée par la valeur des débits mesurés au cours de l'épisode du 3 février 2009.

- Dans le bassin-versant du Crébadès, la relation est moins bien définie. À surfaces drainées équivalentes et pour un même épisode, les débits mesurés restent effectivement très variables. Il paraît donc difficile, au vu des épisodes observés, d'établir une relation permettant d'expliquer les débits par la seule surface amont drainée dans ce bassin. Néanmoins, il semble que pour l'épisode le plus important, celui du 3 novembre 2008, une relation puisse relier la surface drainée aux débits mesurés à partir d'un seuil surfacique voisin de $1000 \mathrm{~m}^{2}$. La valeur de ce seuil nécessite maintenant d'être confirmée par l'étude d'autres événements.

- Une analyse particulière a été menée afin de tenter d'expliquer les débits mesurés inférieurs aux seuils mis en évidence in fig. 3, par d'autres paramètres hydromorphologiques (i.e., ruptures de pente amont ou aval, densité de drainage, indice d'encaissement, etc.) extraits de l'analyse du MNT TIN. Aucun des paramètres étudiés (Maréchal, 2011) ne permet d'établir de relation avec les débits mesurés sur cette plage de surfaces drainées.

- L'observation distribuée des débits lors des épisodes pluvieux a permis de distinguer deux types de réseau : 1) un réseau « principal » dans lequel les débits suivent une relation avec la surface drainée et 2) un réseau « secondaire » dans lequel les débits ne sont ni expliqués par la surface drainée, ni par les paramètres hydromorphologiques étudiés.

Mais la valeur des débits n'est pas le seul témoin de la réaction hydrologique des bassins. Elle peut également être étudiée au regard de l'extension des réseaux en eau et de l'occurrence de leur mise en eau. Les paragraphes suivant s'attachent à étudier le rôle des paramètres morphologiques sur les mécanismes de mise en eau et de maintien des écoulements au sein des réseaux.

\section{Analyse de l'occurrence de mise en eau des drains - Descripteurs morphologiques}

Les campagnes d'observation ont permis de proposer des cartographies de la pérennité des drains sous la forme d'occurrence de mise en eau du réseau hydrographique dans les deux bassins versants expérimentaux. La fig. 5 présente ces cartographies pour les deux bassins versants au regard de celles des réseaux principaux et secondaires déterminés à partir des seuils de surfaces drainées mis en évidence précédemment.

Pour le bassin-versant du Crébadès, dans le sous-bassin nord, les tronçons les plus pérennes constituent le thalweg principal, renforçant l'hypothèse que ce dernier représente à lui seul le réseau principal de ce sous-bassin. Dans le sousbassin sud, le réseau principal est constitué des deux thalwegs principaux, présentant les tronçons les plus fréquemment en eau. Dans le bassin-versant du Cartaou, la situation est plus complexe dans la mesure où, si le thalweg principal est le tronçon le plus fréquemment en eau du bassin, les écoulements de versant, même s'ils drainent des surfaces supérieures à $5000 \mathrm{~m}^{2}$, sont les plus temporaires du bassin. Ces différences de pérennité des réseaux ne peuvent donc être expliquées, pour ce bassin-versant, ni par la surface amont drainée ni par l'appartenance au réseau principal du bassin.

Dans le bassin-versant du Crébadès, si la concordance entre la distinction réseau principal/réseau secondaire et l'occurrence de mise en eau des drains semble établie, cette relation semble donc plus complexe dans le bassin-versant du Cartaou. Cette situation pousse à s'intéresser plus en détail aux liens entre les caractéristiques morphologiques des drains et leur occurrence de mise en eau. La figure 5 fait ainsi état de l'ensemble des occurrences de mises en eau du réseau hydrographique du bassin-versant du Cartaou en fonction de l'indice de pente moyenne amont et de la surface drainée. Sont distingués les trois linéaires principaux du bassin du Cartaou identifiés in fig. 4B. Ils correspondent aux linéaires dont la surface drainée est supérieure à $9000 \mathrm{~m}^{2}$ et les occurrences égales ou supérieures à 5. Le linéaire $n^{\circ} 1$ correspond au thalweg principal (fig. 4B), de l'exutoire du bassin jusqu'à la crête la plus éloignée. Il est représenté par les drains les plus pérennes (7 et 8 fois en eau) et présente un profil particulièrement accidenté, ses pentes moyennes amont variant brutalement tout au long de son parcours. Diminuant dans la partie amont, les pentes augmentent assez nettement à l'aval du drain principal, pour une surface drainée de l'ordre de $100000 \mathrm{~m}^{2}$, pour atteindre finalement

Fig. 5 - Réseaux principaux et secondaires et cartographie de l'occurrence de mise en eau des drains. A : Bassin du Cartaou. A1: appartenance au réseau principal. 1 : réseau principal (drainant une surface $>5000 \mathrm{~m}^{2}$ ) ; 2 : réseau secondaire (drainant une surface $<5000 \mathrm{~m}^{2}$ ). A2 : occurrence de mise en eau des réseaux hydrographiques observés. $B$ : Bassin du Crébadès. 1-8 : occurrences de mise en eau des drains. B1 : appartenance au réseau principal. 1 : réseau principal en eau le 3 novembre 2008 ; 2 : réseau secondaire en eau le 3 novembre 2008. B2 : 1-8 : occurrence de mise en eau des réseaux hydrographiques observés.

Fig. 5 - Main and secondary networks and hydrological occurrences map. A: Cartaou catchment. A1: affiliation to the principal network. 1: main hydrographical network (drainage area $>5000 \mathrm{~m}^{2}$ ); 2 : secondary hydrographical network (drainage area $<5000 \mathrm{~m}^{2}$ ). A2: 1-8: occurrence of water presence in the observed hydrographic network. $B$ : Crébadès catchment. B1: affiliation to the principal network. 1: main hydrographical network flooded the 3rd November 2008; 2: secondary hydrographical network flooded the 3rd November 2008. B2: 1-8: occurrence of water presence in the observed hydrographic network. 


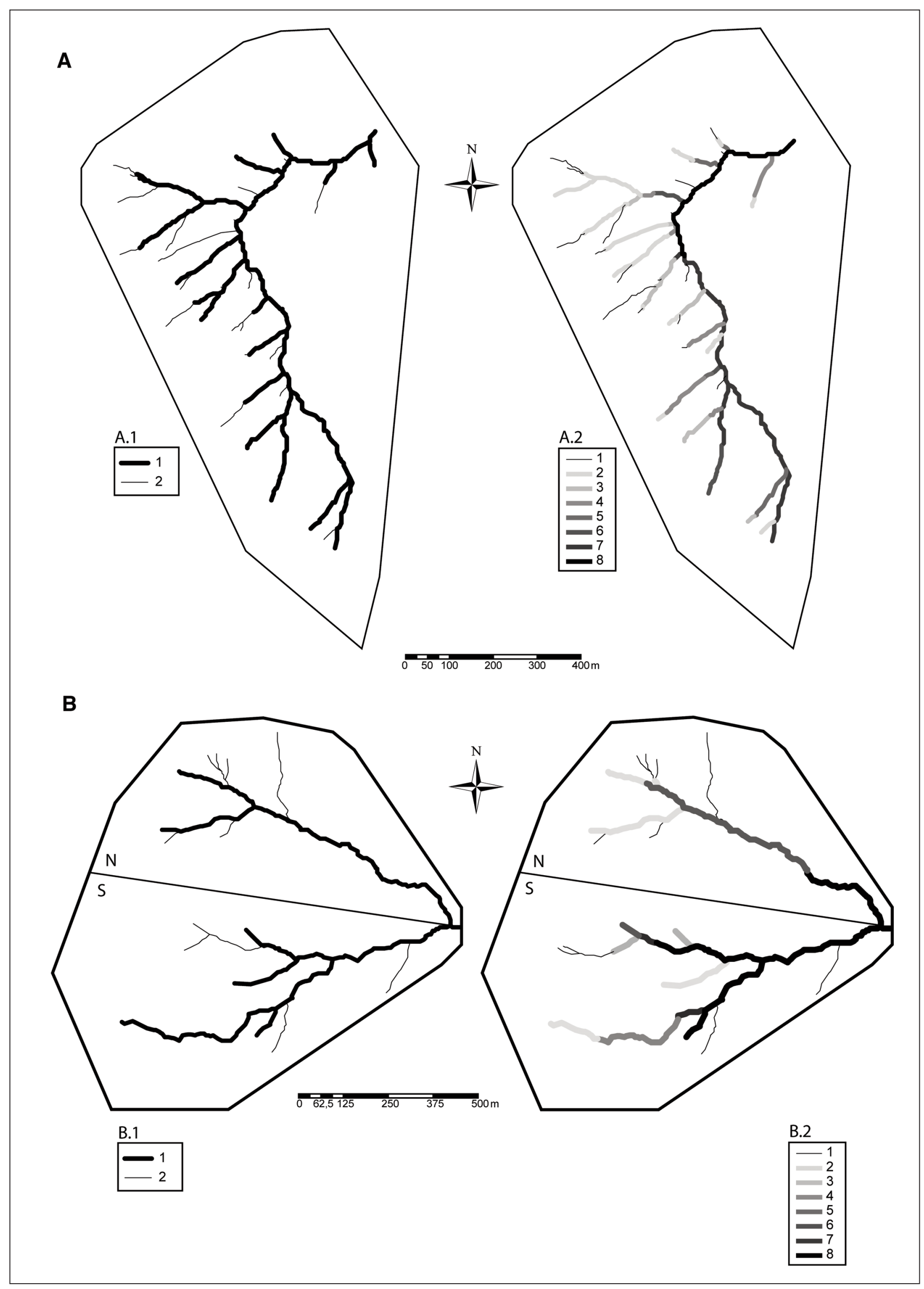


la pente moyenne du bassin, de l'ordre de $50 \%$. Ce seuil correspond à la zone de confluence des deux drains principaux représentés par les linéaires $n^{\circ} 1$ et 2 . Le linéaire $n^{\circ} 2$ correspond à l'affluent le plus important du thalweg principal du bassin (fig. 4B). De la même façon, sa représentation est chaotique au regard des différentes valeurs de pente qui oscillent avec importance de l'amont vers l'aval. Il présente des pentes plus importantes (55-60\%) que le thalweg principal. Enfin, le linéaire $n^{\circ} 3$ correspond à un court linéaire observé 5 fois en eau, situé à l'amont du versant sud (fig. 4B). Il présente des pentes équivalentes à celles du thalweg principal en haut du même versant.

Sur la fig. 5, il est difficile de séparer le linéaire du thalweg principal des réseaux de versants venant l'alimenter. L'organisation du réseau de ce bassin est en partie responsable de cette confusion. En effet, le drain principal remontant très haut sur les versants et les drains de versant étant répartis tout le long du parcours du drain principal, les caractéristiques de pente et de surface drainée du thalweg principal et des drains de versant sont confondues. Seuls quelques linéaires sont clairement identifiables (cercles et lignes in fig. 5). En effet, les différentes occurrences de mise en eau des drains les plus temporaires ne semblent pas être fonction des surfaces drainées, allant de $800 \mathrm{~m}^{2}$ à $80000 \mathrm{~m}^{2}$ (les drains observés 2 fois en eau sont par exemple repré- sentés sur la quasi-totalité de la plage des surfaces drainées). De même, les pentes moyennes amont sont équivalentes entre les différentes occurrences de mise en eau. Cette organisation du réseau secondaire vient confirmer les observations effectuées auparavant. Ce réseau est en effet composé de drains répartis sur l'ensemble des versants ouest, relativement similaires du point de vue topographique. Ils favorisent des écoulements de surface régulièrement distribués mais de pérennités différentes. C'est pourquoi les caractéristiques morphologiques présentées in figure 6 ne semblent pas entretenir de relations évidentes avec la temporalité des différents drains.

\section{Discussion}

Cette étude a permis de distinguer deux types de réseaux : 1) le réseau principal dans lequel les débits suivent une relation mathématique (ici puissance) avec la surface drainée et 2) le réseau secondaire dans lequel les débits ne sont ni expliqués par la surface drainée, ni par les paramètres morphologiques étudiés. Il semble en effet que dans le réseau principal, la seule surface drainée est capable de résumer l'ensemble des interactions complexes responsables de la production des débits mesurés au sein de celui-ci. Au contraire, au sein du réseau secondaire, les débits mesurés ne semblent pas avoir de rela-

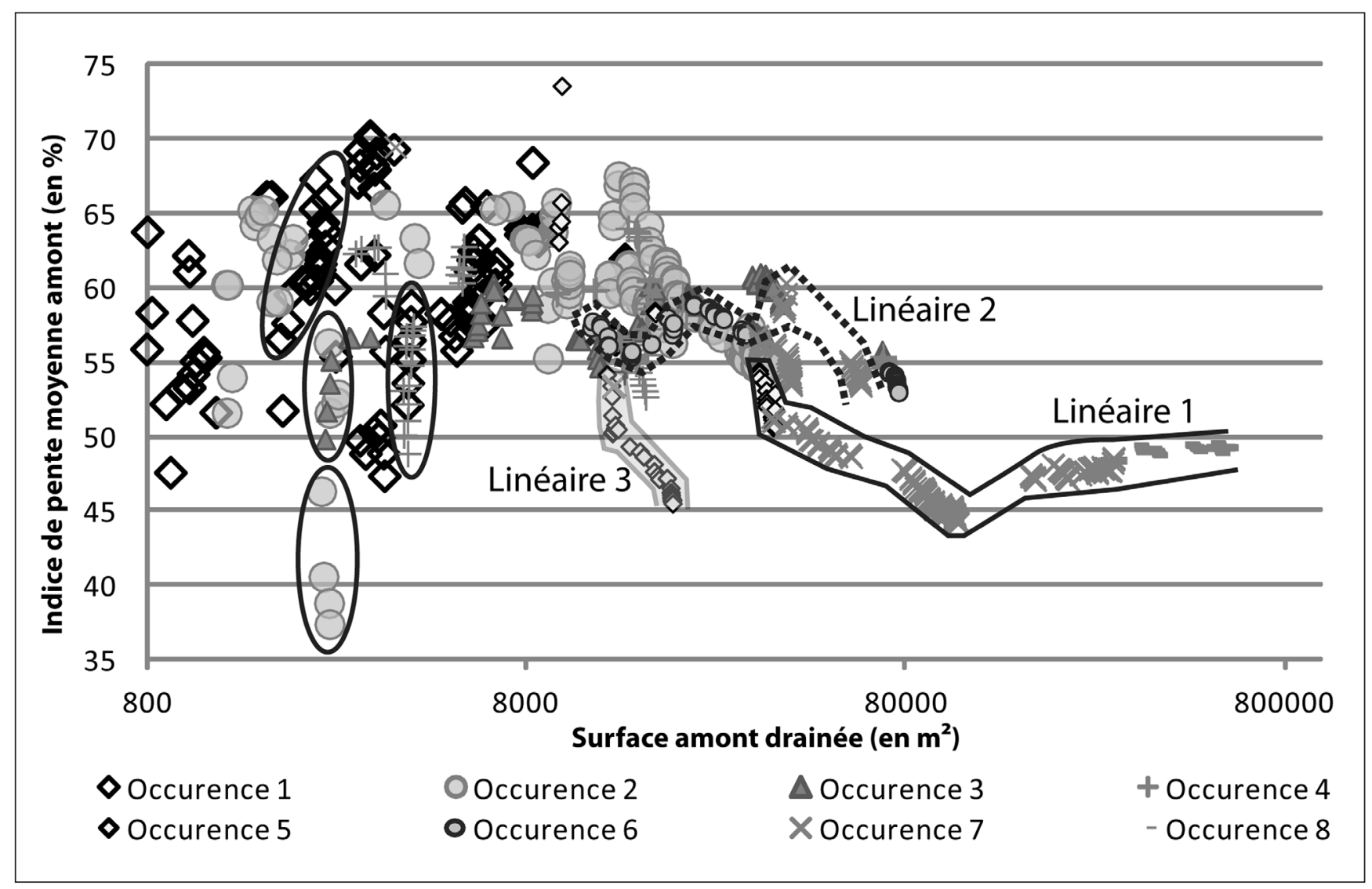

Fig. 6 - Surface amont drainée et pente moyenne amont des différentes occurrences de mises en eau du réseau hydrographique observé du bassin du Cartaou.

Fig. 6 - Drainage area and mean slope in the upstream part of hydrologically active networks in the Cartaou catchment. 


\begin{tabular}{|c|c|c|}
\hline Objets d'étude & Crébadès & Cartaou \\
\hline $\begin{array}{l}\text { Facteur explicatif de la valeur des débits } \\
\text { observés }\end{array}$ & $\begin{array}{l}\text { Surface drainée uniquement sur } \\
\text { l'évènement le plus important à partir de } \\
1000 \mathrm{~m}^{2}\end{array}$ & $\begin{array}{l}\text { Surface drainée pour tous les épisodes à } \\
\text { partir de } 5000 \mathrm{~m}^{2}\end{array}$ \\
\hline $\begin{array}{l}\text { Réseau systématiquement en eau } \\
\text { (Maréchal, 2011) }\end{array}$ & $\begin{array}{l}\text { Seuil de surface drainée de } 260000 \mathrm{~m}^{2} \text { sur } \\
\text { la période observée (2008-2010) }\end{array}$ & $\begin{array}{l}\text { Seuil de surface drainée de } 40000 \mathrm{~m}^{2} \text { sur la } \\
\text { période observée (2008-2010) }\end{array}$ \\
\hline $\begin{array}{l}\text { Surface minimale pour l'initiation des } \\
\text { écoulements dans les drains (Maréchal, } \\
\text { 2011) }\end{array}$ & $\begin{array}{l}\text { Seuil de surface drainée : } 150 \mathrm{~m}^{2} \text { sur la } \\
\text { période observée }(2008-2010)\end{array}$ & $\begin{array}{l}\text { Seuil de surface drainée : } 400 \mathrm{~m}^{2} \text { sur la } \\
\text { période observée }(2008-2010)\end{array}$ \\
\hline Réseau principal & Contraint aux talwegs principaux & $\begin{array}{l}\text { Thalwegs principaux et chevelus de } \\
\text { versants }\end{array}$ \\
\hline Sols & $\begin{array}{l}\text { De type ranker (de } 30 \text { à } 50 \mathrm{~cm} \text { ), densité } \\
\text { apparente faible, porosité importante. } \\
\text { Certains versants ne présentent pas de } \\
\text { sols mais des affleurements de schistes } \\
\text { fracturés. }\end{array}$ & $\begin{array}{l}\text { De type ranker (de } 30 \mathrm{~cm} \text { à } 1 \mathrm{~m} \text { ), densité } \\
\text { apparente faible, porosité importante }\end{array}$ \\
\hline Substratum & $\begin{array}{l}\text { Schistes avec présence d'une épaisseur de } \\
\text { schistes altérés. Affleurement de schistes } \\
\text { fracturés sur certains versants. }\end{array}$ & $\begin{array}{l}\text { Granite, avec présence d'arène granitique } \\
\text { sur une épaisseur importante sur certains } \\
\text { versants }(>2 \mathrm{~m})\end{array}$ \\
\hline Processus de genèse des crues & $\begin{array}{l}\text { Prépondérance de l'écoulement de sub- } \\
\text { surface dans les premiers centimètres du } \\
\text { sol et dans la zone altérée (écoulement en } \\
\text { macropores). Cet écoulement est } \\
\text { grandement favorisé par la pente des } \\
\text { versants. Sur ce bassin, l'hypothèse d'un } \\
\text { temps de séjour court de l'eau dans le sol } \\
\text { (i.e., exfiltration rapide de l'eau vers les } \\
\text { thalwegs) a été proposée par plusieurs } \\
\text { auteurs suite à des expérimentations et } \\
\text { observations de terrain (Ayral, } 2005 \text {; Ayral } \\
\text { et al., 2005). }\end{array}$ & $\begin{array}{l}\text { Ecoulement de sub-surface qui existe mais } \\
\text { qui reste très limité pour les versants qui } \\
\text { présentent des arènes granitiques. Sur ce } \\
\text { bassin-versant, un processus de genèse } \\
\text { des crues par saturation en bas de versant } \\
\text { est privilégié par de nombreux auteurs suite } \\
\text { à des travaux expérimentaux et de } \\
\text { modélisation (Ayral et al., } 2005 \text {; Bouvier et } \\
\text { al., } 2006 \text {; Martin et al., } 2008 \text {; Tramblay et } \\
\text { al., 2010; Maréchal, } 2011 \text {; Tramblay et al., } \\
\text { 2011). }\end{array}$ \\
\hline
\end{tabular}

Tab. 6 - Résumé des fonctionnements différenciés des deux bassins versants pilotes.

Tab. 6 - Summary of the differentiated behaviours for the two experimental catchments.

tion claire et établie avec les caractéristiques morphologiques étudiées dans ce travail. Liés à des processus complexes au sein des versants, dans les sols et le sous-sol, il est probable que les débits soient moins influencés par des paramètres caractérisant la morphologie de surface du bassin et des réseaux que par des paramètres liés à la production des débits au sein des versants. On s'approche donc ici de l'évaluation quantitative d'une « limite » pertinente pour la modélisation hydrologique, à savoir celle entre la production (i.e., le versant) et le transfert (i.e., le thalweg). Il convient bien évidemment de relativiser ces premiers résultats au regard du peu d'événements étudiés dans les deux bassins versants. De nouvelles observations doivent donc être acquises avant de pouvoir préciser les valeurs de ces seuils et éventuellement généraliser leurs utilisations à d'autres bassins versants. Les résultats sont ici discutés successivement au regard 1) des différences observées entre les deux bassins versants et 2) de la méthodologie hydrométrique distribuée mise en place.

\section{Comparaison des fonctionnements des deux bassins expérimentaux}

Le tableau 6 résume les différents seuils mis en évidence dans cette étude au regard des caractéristiques des deux bas- sins versants et des processus hydrologiques de genèse des crues identifiés dans la littérature (Ayral et al., 2005 ; Bouvier et al., 2006 ; Marchandise, 2007 ; Martin et al., 2008 ; Tramblay et al., 2010 ; Maréchal, 2011 ; Tramblay et al., 2011).

Les différences observées sur les seuils d'initiation du réseau principal entre les deux bassins semblent pouvoir être expliquées par les types d'écoulements majoritaires des deux bassins expérimentaux. Dans le bassin-versant du Cartaou, le processus identifié pour expliquer la genèse des écoulements lors des crues semble correspondre à un fonctionnement de type saturation en bas de versant (tab. 6). Ce fonctionnement implique donc a priori que la production des écoulements soit contrôlée par la profondeur du sol et des couches altérées jusqu'à saturation du profil, cette saturation intervenant en bas de versant et donc à des surfaces relativement conséquentes (un seuil de $5000 \mathrm{~m}^{2}$ a été identifié dans ces travaux). A contrario, les écoulements dans le sol ou au sein des fracturations des schistes observés dans le bassin versant du Crébadès (Ayral et al., 2005 ; Maréchal, 2011) devraient être moins soumis à des effets de seuil induits par un fonctionnement de type saturation d'un profil de sol mais plutôt contrôlé par des phénomènes d'exfiltration, les volumes écoulés ainsi seraient donc plus rapidement fonction de la surface amont drainée (1 $000 \mathrm{~m}^{2}$ obser- 
vé pour un événement). Les processus ici évoqués pour proposer une première explication des différences de seuils de surface drainée obtenus pour délimiter le réseau principal et le réseau secondaire sont en cohérence avec la surface minimale pour l'apparition de l'eau dans les drains (tab. 6) lors des épisodes pluvieux observés dans les deux bassins (Maréchal, 2011). Cette surface est en effet de $150 \mathrm{~m}^{2}$ pour le bassin du Crébadès et de $400 \mathrm{~m}^{2}$ pour le bassin du Cartaou.

En ce qui concerne la pérennité du réseau en eau, le bassin du Cartaou accueille un réseau permanent beaucoup plus développé (à partir d'une surface drainée de $40000 \mathrm{~m}^{2}$ ) que celui du Crébadès (à partir d'une surface drainée 260000 m²). Il présente un thalweg principal aux écoulements quasi-permanents, dans lequel les débits sont pour tous les épisodes fonction de la surface amont drainée, et se distinguant clairement des réseaux plus temporaires de versants qui l'alimentent tout au long de son parcours. La présence d'arènes granitiques dans le bassin doit être un élément central pour expliquer l'alimentation pérenne de ce réseau principal. Le bassin du Crébadès, qui ne dispose pas de capacité de stockage équivalente à celle du Cartaou, présente un réseau fonctionnant temporairement, certains drains étant asséchés quasiment toute l'année. Ces drains sont toutefois particulièrement réactifs lors des épisodes pluvieux.

\section{De l'intérêt du suivi spatialisé des réponses hydrologiques des bassins}

La dynamique des débits à l'exutoire ne suffit pas à rendre compte de la complexité de l'agencement des processus hydrologiques responsables de la mise en eau et de la pérennité des écoulements surfaciques au sein des bassins versants. A l'inverse, cette étude, qui étudie les variations spatiales des débits au sein de l'ensemble du réseau hydrographique en eau, permet d'approcher les variations spatio-temporelles des processus hydrologiques en jeu au cours des épisodes observés à l'intérieur même des bassins. En outre, les débits ne représentent pas la seule information permettant de caractériser la réaction des bassins. La localisation des départs d'écoulements, l'extension des réseaux et la pérennité de leurs écoulements sont également des paramètres utiles et pertinents pour l'analyse complète des réponses hydrologiques d'un bassin aux épisodes pluvieux. Un suivi systématique et spatialisé de ces réponses permet de rendre compte de la diversité des mécanismes responsables de la variété des réponses hydrologiques observées (Maréchal, 2011).

\section{Limites du suivi spatialisé}

Le suivi des réponses hydrologiques des bassins reste soumis aux contraintes imposées par le terrain. Tout d'abord, certains drains, parfois inaccessibles, ne peuvent être régulièrement suivis. En outre, les observations, pour des raisons d'incertitude météorologique, ne peuvent conduire systématiquement aux mêmes moments hydrologiques des différents épisodes, limitant les comparaisons inter-événementielles. Enfin, les valeurs des débits mesurés sont soumises aux incertitudes techniques de mesures. En effet, même si la méthode de jaugeage par dilution de sel est particulièrement adaptée aux écoulements torrentiels (André et al., 1976 ; Musy, 2005), de nombreuses incertitudes subsistent quant à la valeur précise des débits d'un cours d'eau. Enfin, l'investissement important que nécessite ces suivis, en vue d'obtenir la vision la plus complète possible des variations spatio-temporelles des réponses hydrologiques d'un bassin, limite toute généralisation à des bassins de grande superficie. Cependant, la compréhension des processus hydrologiques, facilitée à cette échelle de travail, peut permettre, à terme, la généralisation des processus à d'autres bassins non jaugés et apportent des informations pertinentes pour la modélisation hydrologique de leurs réponses.

\section{Intérêt de l'étude de l'origine morphologique des réponses hydrologiques}

Les caractéristiques morphologiques étudiées dans ce travail sont utilisées comme des indicateurs des réponses hydrologiques potentielles des bassins expérimentaux. Bien qu'ils soient influencés par l'évolution des reliefs, liée notamment aux phénomènes érosifs, ces paramètres morphologiques sont relativement stables dans le temps. Ils influent sur les vitesses de ruissellement, les potentialités d'exfiltration ainsi que sur le déclenchement des écoulements et leur pérennité. Ils permettent donc de spatialiser les caractéristiques de bassin pouvant jouer un rôle dans les variabilités spatio-temporelles des réponses hydrologiques observées. À ce titre, ils sont de bons indicateurs des réponses hydrologiques potentielles des bassins. Ils peuvent donc aider à la prédiction des types de réponses hydrologiques générées par des pluies événementielles sur des bassins dont seules les caractéristiques géomorphologiques sont disponibles sous la forme d'un MNT. Cependant, la généralisation des mécanismes contrôlant les réponses hydrologiques des bassins reste difficilement réalisable. Essentiellement contrôlée par des mécanismes locaux de production des écoulements, les réponses hydrologiques des bassins doivent être étudiées au regard de paramètres complémentaires pouvant aider à la généralisation des processus. La présente étude met ainsi en avant le rôle prépondérant des sols et de leurs caractéristiques sur la mise en eau, le maintien et la pérennité des écoulements au sein des réseaux.

Cette étude a permis la distinction entre des réseaux aux fonctionnements liés à des processus de production des écoulements au sein des versants (réseau secondaire) et des réseaux davantage soumis aux lois de l'hydraulique (réseau principal). Le lien avec leur déclenchement et leur occurrence de mise en eau reste toutefois difficile à établir (notamment dans le bassin du Cartaou), même si son étude se révèle riche d'enseignements pour la compréhension des processus hydrologiques à l'intérieur des bassins versants.

Aujourd'hui, l'extension des réseaux utilisée dans les modèles hydrologiques pour le transfert des écoulements de surface ne s'appuient sur aucune analyse de fonctionnement hydrologique mais sur une extension définie a priori et utilisant des critères de surfaces minimum d'initiation des écoule- 
ments, pourtant variables entre les différentes zones d'un même bassin ou pour différents épisodes. En étudiant le fonctionnement hydrologique des réseaux et les différents critères morphologiques responsables des débits mesurés en leur sein, l'approche suivie dans ce travail permet de définir une extension de réseau au sein duquel les débits sont modélisables à partir de paramètres simples issus de l'analyse de MNT, et ceci pour l'ensemble des événements. S'il semble impossible de modéliser les débits dans le reste du réseau, il participe néanmoins à la production des débits modélisables au sein du réseau principal. C'est pourquoi il apparaît nécessaire d'envisager une étude de sensibilité des modèles hydrologiques distribués utilisant uniquement les réseaux principaux identifiés dans les deux bassins.

\section{Conclusions}

L'étude de l'origine morphologique des débits et de la pérennité des drains en eau a permis de fournir des clés de compréhension des mécanismes responsables des fonctionnements différenciés des différents réseaux (principaux ou secondaires) des bassins. Ces deux types de réseau se distinguent par leur fonctionnement différencié au regard des caractéristiques morphologiques. Les réseaux secondaires occupent en majorité les versants, sur des pentes importantes, et drainent des surfaces plus limitées que le réseau principal. Ils semblent davantage soumis aux processus de production des débits au sein des versants, contrôlés par des effets de seuils. Le réseau principal des bassins, dont la morphologie est plus marquée et la pente plus modérée, est davantage soumis aux lois de l'hydraulique majoritairement contrôlées par la surface drainée. L'influence de la morphologie semble ainsi limitée au réseau principal des bassins. En dehors, elle paraît négligeable au regard des caractéristiques pédologiques et géologiques responsables de la production des écoulements au sein des versants. En outre, la distinction entre ces deux types de fonctionnement varie dans l'espace et le temps. Dans le bassin du Crébadès, l'étude a ainsi montré une extension variable du réseau principal en fonction des épisodes de crue. A partir de ce constat, il apparaît primordial pour la modélisation hydrologique de prendre en compte une extension variable des réseaux hydrographiques en fonction des épisodes de crue, en utilisant les caractéristiques morphologiques indicatrices des réponses hydrologiques des bassins. La présente étude propose une approche originale permettant de suivre et de mieux comprendre les dynamiques spatio-temporelles des réponses hydrologiques de petits bassins amont cévenols sous épisode pluvieux. Par l'étude des relations statistiques entre des indicateurs morphologiques spatialisés issus des nouvelles techniques spatiales et les variations spatio-temporelles des réponses hydrologiques provenant de mesures multi-locales au sein des bassins expérimentaux, elle a permis d'analyser l'étendue de l'influence de la morphologie sur le fonctionnement hydrologique des bassins versants étudiés. Si la poursuite des observations paraît nécessaire pour affiner les seuils de surface drainée proposés, ce travail représente une première étape indispensable vers le développement futur de modèles hydrologiques spatialisés basés sur des indicateurs des réponses hydrologiques potentielles : les caractéristiques morphologiques des bassins et de leurs réseaux.

\section{Remerciements}

Les auteurs tiennent à remercier : le CNES (Centre national d'études spatiales) pour son soutien à ce projet de recherche; l'IFR ILEE (Institut languedocien de recherche sur l'eau et l'environnement) qui a également soutenu ce programme au travers du projet MUSHYCAL (Mesures hydrologiques multilocales par capteurs hydrométriques légers) ; Hervé Ayroles de l'IMFT (Institut de mécanique des fluides de Toulouse) pour le développement, la mise au point et la fabrication de capteurs limnimètres capacitifs. Les auteurs tiennent également à remercier l'ANR SYSCOMM qui, dans le cadre du projet FLASH, a permis le développement de la démarche d'observations hydrologiques distribuées. Enfin, les auteurs tiennent à remercier les relecteurs anonymes de cet article, dont les corrections et commentaires ont permis d'améliorer grandement la qualité du papier présenté, et Norma Williston (département Langues de l'Ecole des Mines d'Alès) pour sa relecture des textes en anglais.

\section{Références}

Ambroise B. (1998) - Genèse des débits dans les petits bassins versants ruraux en milieu tempéré : processus et facteurs. Revue des sciences de l'eau, 4, 475-495.

André H., Audinet M., Mazeran G., Richer C. (1976) - Hydrométrie pratique des cours d'eau. Eyrolles, Paris, 59 p.

Ayral P.-A. (2005) - Contribution à la spatialisation du modèle opérationnel de prévision des crues éclair ALHTAÏR. Approches spatiale et expérimentale. Application au bassin versant du Gardon d'Anduze. Thèse de doctorat en Biosciences de l'Environnement, Chimie et Santé, université de Provence (Aix-Marseille I), $311 \mathrm{p}$.

Ayral P.-A., Sauvagnargues-Lesage S., Bressand F. (2005) Contribution à la spatialisation du modèle opérationnel de prévision des crues éclair ALHTAÏR. Etudes de Géographie Physique, 32, 75-97.

Bhadra A., Panigrahy N., Singh R., Raghuwanshi N.S., Mal B.C., Tripathi M.P. (2008) - Development of a geomorphological instantaneous unit hydrograph model for scantily gauged watersheds. Environmental Modelling \& Software 23, 1013-1025.

Bouvier C., Ayral P.A., Brunet P., Crespy A., Marchandise A., Martin C. (2006) - Recent advances in rainfall-runoff modeling: extrapolation to extreme floods in southern France. Observing and modelling exceptional floods and rainfalls, Proceedings of the AMHY-FRIEND International Workshop on Hydrological Extremes, held at University of Calabria, Cosenza (Italy), May 3-4, 2006.

Chevalier P. (1983) - L'indice des précipitations antérieures. Evaluation de l'humectation des sols des bassins versants représentatifs. Cahier de l'ORSTOM, série Hydrologie, 20, 3-4.

Delrieu G., Ducrocq V., Gaume E., Nicol J., Payrastre O., Yates E., Kirstetter P.E., Andrieu H., Ayral P.-A., Bouvier C., Creu- 
tin J.-D., Livet M., Anquetin S., Lang M., Neppel L., Obled C., Parent-du-Chatelet J., Saulnier G.M., Walpersdorf A., Wobrock W. (2005) - The catastrophic flash-flood event of 8-9 September 2002 in the Gard region, France: a first case study for the Cévennes-Vivarais Mediterranean Hydrometeorological Observatory. Journal of Hydrometeorology 6, 34-52.

De Vries H., Becker T., Eckhardt B. (1994) - Power law distribution of discharge in ideal networks. Water Resources Research 30, 3541-3543.

Gaume E. (2002) - Eléments d'analyse des crues éclair. Thèse de doctorat, Ecole Nationale du Génie Rural des Eaux et des Forêts, 359 p.

Gaume E., Bouvier C. (2004) - Analyse hydro-pluviométrique des crues du Gard et du Vidourle des 8 et 9 septembre 2002. La Houille Blanche, 6, 99-106.

Gupta V.K., Waymire E., Wang C.T. (1980) - A representation of an instantaneous unit hydrograph from geomorphology. Water Resources Research 16, 855-862.

Horton R.E. (1932) - Drainage basin characteristics. Eos Transactions, American Geophysical Union 13, 350-361.

Kumar R., Chatterjee C., Singh R.D., Lohani A.K., Kumar S. (2007) - Runoff estimation for an ungauged catchment using geomorphological instantaneous unit hydrograph (GIUH) models. Hydrological Processes 21, 1829-1840.

Lee K.T., Chen N.-C., Chung Y.-R. (2008) - Derivation of variable IUH corresponding to time-varying rainfall intensity during storms. Journal of Hydrological Sciences 53, 323-337.

Mantilla R., Gupta V.K., Mesa O.J. (2006) - Role of coupled flow dynamics and real network structures on Hortonian scaling of peak flows. Journal of Hydrology 322, 155-167.

Marchandise A. (2007) - Modélisation hydrologique distribuée sur le Gardon d'Anduze ; étude comparative de différents modèles pluie-débit, extrapolation de la normale à l'extrême et tests d'hypothèses sur les processus hydrologiques. Thèse de doctorat en Terre, Eau, Espace, université Montpellier 2, 300 p.

Maréchal D. (2011) - Du drain potentiel au drain réel : utilisation de données satellitales à très haute résolution pour l'étude de l'origine géomorphologique des chemins de l'eau sur des bassins versants soumis aux crues éclair. Thèse de l'Ecole doctorale des Mines de Saint-Etienne, 313 p.

Maréchal D., Puech C., Martin C., Bailly J.-S., Ayral P.-A., Ayroles H. (2009) - Use of new, diverse and widely distributed in situ hydrological measurements to improve catchment flow dynamics understanding. European Geosciences Union, General Assembly, Vienne, Austria, 19-24 April 2009.

Maréchal D., Ayral P.-A., Bailly J.-S., Puech C., Sauvagnargues-Lesage S. (2010) - ExtracTIN: An original algorithm for automatic hydrogeomorphological features extraction using remote sensing data. BALWOIS - Water Observation and Information System for Decision Support, Ohrid Republic of Macedonia, 25-29 May 2010.

Martin C. (2006) - Les systèmes de terrasses cévenols. Exemple de la vallée obscure et du vallon du Rouquet. Etude de géogra- phie physique. Projet Interreg IIIB SUDOE «TERRISC ». UMR Espace, CNRS, Avignon, 153 p.

Martin C., Didon-Lescot J.-F., Jolivet J. (2008) - Réflexion sur le rôle hydrologique des aménagements anciens des versants et des thalwegs : l'exemple des Cévennes. Les eaux : problèmes de gestion et de qualité. Travaux du laboratoire de Géographie Physique Appliquée, 26, 31-40.

Martz L.W., Garbrecht J. (1995) - Automated recognition of valley lines and drainage networks from grid digital elevation models: a review and a new method. Journal of Hydrology, 167, 393-396.

Moore I.D., Foster G.R. (1990) - Hydraulics and overland flow. In Anderson M.G., Burt T.P .(Eds.), Process Studies in Hillslope Hydrology. Wiley, New York, 215-252.

Moussa R. (1991) - Variabilité spatio-temporelle et modélisation hydrologique. Application au bassin du Gardon d'Anduze. Thèse de doctorat. Université des Sciences et Techniques du Languedoc (Montpellier 2), $259 \mathrm{p}$.

Moussa R. (2009) - Definition of new equivalent indices of HortonStrahler ratios for the derivation of the geomorphological instantaneous unit hydrograph. Water Resources Research, 45, 24 p.

Musy A. (2005) - Hydrologie générale. Cours de l'Ecole Polytechnique de Lausanne. http://echo2.epfl.ch/e-drologie/

Rodriguez-Iturbe I., Valdès J.B. (1979) - The geomorphologic structure of hydrologic response. Water Resources Research, 15, 1409-1419.

Séranne M., Camus H., Lucazeau F., Barbarand J., Quinif Y. (2001) - Surrection et érosion polyphasées de la bordure cévenole. Un exemple de morphogenèse lente. Bulletin de la Société Géologique de France, 173, 2, 97-112.

Singh P.K., Bhunya P.K, Mishraa S.K., Chaube U.C. (2007) An extended hybrid model for synthetic unit hydrograph derivation. Journal of Hydrology 336, 247-360.

Tarboton D.G. (1997) - A new method for the determination of flow directions and upslope areas in grid digital elevation models. Water Resources Research 33, 309-319.

Toukourou M., Johannet A., Dreyfus G., Ayral P.-A. (2009) Rainfall-Runoff modeling for flash floods: the case of "Cévenol flash floods". Journal of Applied Intelligence, $22 \mathrm{p}$.

Tramblay Y., Bouvier C., Matin C., Didon-Lescot J.-F., Todorovik D., Domergue J.-M. (2010) - Assessment of initial soil moisture conditions for event-based rainfall-runoff modelling. Journal of Hydrology 387, 176-187.

Tramblay Y., Bouvier C., Ayral P.-A., Marchandise A. (2011) Impact of rainfall spatial distribution on rainfall-runoff modeling efficiency and initial soil moisture conditions estimation. Natural Hazards and Earth System Sciences, 11, 157-170.

White A.B., Kumar P., Saco P.M., Rhoads B.L., Yen B.C. (2004) Hydrodynamic and geomorphologic dispersion: Scale effects in the Illinois River Basin. Journal of Hydrology 288, 237-257.

Article soumis le 16 mai 2011, accepté le 15 décembre 2011. 